\title{
Based on Network Pharmacology and Molecular Docking Technology, the Mechanism of Folium Sennae, Aloe Combined with Panax Quinquefolium in Treating Functional Constipation Was Investigated
}

\author{
Yanrong Zhan ${ }^{1}$, Yaping Cui ${ }^{2, *}$, Yang Liu ${ }^{1}$, Jiyun $\mathrm{Wu}^{1}$, Yan Ou${ }^{1}$, Hua Jiang ${ }^{2}$ \\ ${ }^{1}$ Shaanxi University of Chinese Medicine, Xianyang, Shaanxi 712000, China. \\ ${ }^{2}$ Affiliated Hospital of Shaanxi University of Chinese Medicine, Xianyang, Shaanxi 712000, China.
}

\begin{abstract}
How to cite this paper: Yanrong Zhan, Yaping Cui, Yang Liu, Jiyun Wu, Yan Ou, Hua Jiang. (2021) Based on Network Pharmacology and Molecular Docking Technology, the Mechanism of Folium Sennae, Aloe Combined with Panax Quinquefolium in Treating Functional Constipation Was Investigated. International Journal of Clinical and Experimental Medicine Research, 5(4), 466-479.

DOI: $10.26855 /$ ijcemr.2021.10.008
\end{abstract}

Received: August 8, 2021

Accepted: August 31, 2021

Published: September 28, 2021

*Corresponding author: Yaping Cui, Affiliated Hospital of Shaanxi University of Chinese Medicine, Xianyang, Shaanxi 712000, China.

Email: 913141097@qq.com

\begin{abstract}
Objective: To explore the mechanism of folium sennae, aloe combined with panax quinquefolium in treating functional constipation (FC) based on network pharmacology and molecular docking method. Methods: Retrieval of all chemical components and action targets of folium sennae, aloe combined with panax quinquefolium using Traditional Chinese Medicine Systems Pharmacology Database and Analysis Platform (tcmsp). Relevant targets for functional constipation were accessed through Gencards, OMIM database, and first-line Western drug targets for the treatment of FC were searched through the drugbank database as a supplement. The STRING data platform was used to construct the interaction network of potential target proteins. Meanwhile, GO (gene ontology) enrichment analysis and KEGG (Kyoto Encyclopedia of Genes and Genomes) signaling pathway data were obtained, and the mechanism of action was analyzed and predicted. The PPI network topology was analyzed by Cytoscape 3.8.0 software, and the network diagram of "component-target-pathway" of FC in the three-drug combination treatment was constructed. Autodock Vina was used for molecular docking to further verify the accuracy of network pharmacological analysis of folium sennae, aloe combined with panax quinquefolium in the treatment of functional constipation. Results: The core active compounds of folium sennae, aloe combined with panax quinquefolium for the treatment of functional constipation are quercetin, kaempferol, $\beta$-Carotene and ginsenoside F2, and the core targets include PTGS2, MAPK1, Jun, VEGFA,IL6, etc. KEGG pathway enrichment analysis showed that the key targets of folium sennae, aloe combined with panax quinquefolium in the treatment of FC were mainly enriched in TNF signaling pathway, IL-17 signaling pathway, VEGF signaling pathway, MAPK signaling pathway, PI3K-Akt signaling pathway, etc. Molecular docking results verified that the average value of binding energy for each compound docking to the core target was less than $-5 \mathrm{kcal} / \mathrm{mol}$ with a good binding activity. Conclusion: This study preliminarily revealed the synergistic effect of panax quinquefolium combined with folium sennae and aloe in the treatment of function-
\end{abstract}


al constipation, and also showed that the combination use of three drugs have the characteristics of multi-component, multi-target, and multi pathway, which laid a foundation for further study of its mechanism of action.

\section{Keywords}

Folium Sennae, Aloe, Panax Quinquefolium, Functional Constipation, Network Pharmacology

\section{Introduction}

Functional constipation (FC) is a kind of constipation caused by exclusion of secondary causes, such as neurogenesis, systemic diseases, intestinal organic lesions and drugs, etc. [1]. According to the diagnostic criteria of Rome IV functional constipation, the symptoms must include two or more of the following: (1) More than 1/4 of the stool or weeks of difficult defecation. (2) dry and hard defecation. (3) feeling of unfulfilled defecation. (4) feeling of anorectal blockage during defecation. (5) need manipulative assisted defecation. (6) defecation less than 3 times per week [2]. In recent years, epidemic epidemiology shows that the total prevalence of functional constipation in children and adults is about $9.5 \%$ [3], and the prevalence rate in adults is $14 \%$, with more women than men [4]. FC is a serious threat to human physical and mental health. Long-term constipation can cause anorectal diseases, gastrointestinal nerve dysfunction, induce and aggravate various cardiovascular and cerebrovascular diseases, and even induce colorectal cancer, hepatic encephalopathy, etc. [5]. The pathogenesis of FC is mainly related to colorectal motility disorders, reduced pelvic floor function, psychosocial factors and abnormal gastrointestinal regulation function [6]. Currently, treatment methods for FC include drug therapy, traditional Chinese medicine technology therapy, enema therapy, biofeedback therapy and surgical therapy [7]. Drug therapy is the most commonly used method in clinical practice, including western medicine, Traditional Chinese medicine and Chinese patent medicine.

Traditional Chinese medicine plays an irreplaceable role in the treatment of functional constipation. Purgative drugs represented by folium sennae and aloe are frequently used in clinical practice. Both folium sennae and aloe are bitter and cold, and both belong to the large intestine meridian, which has laxative efficacy and can be used in the syndrome of heat accumulation, constipation and abdominal pain [8]. However, folium sennae and aloe are the products for relieving the bitter cold, so it is not suitable to be taken for a long time. Taking more than one dose will easily damage the healthy qi and Yin, and easily cause dependence. Panax quinquefolium replenishes qi and Yin, clearing heat and producing fluid [8], and can counteract the side effects of folium sennae and aloe, such as damaging the spleen and stomach due to the bitter cold. The combined use of the three drugs can improve intestinal peristalsis and promote the repair of colonic neurons [9], and at the same time can improve the ecological balance in the intestinal tract and restore the homeostasis between various bacterial communities [10]. Yet studies have reported the mechanism of action of three drugs used in combination treatment of functional constipation, this study based on the network pharmacology method [11] predict senna joint panax quinquefolium, aloe ingredient for FC and mechanism of action, and will receive compounds and key target protein, to verify that the network pharmacology method [12] the accuracy of the results, so as to provide clues for the research and development of new Chinese medicine.

\section{Materials and methods}

\subsection{Collection of active components and target proteins from folium sennae, aloe and panax quinquefo- lium}

Using Chinese medicine pharmacology system analysis platform (TCMSP, https://tcmspw.com/tcmsp.php) [13] retrieve senna, aloe and panax quinquefolium compounds. OB (Oral bioavailability) $\geq 30 \%$ and DL (drug like) $\geq 0.18$ were used as the screening conditions for active ingredients, and the effective compound targets were searched through TCMSP database. Use the PubChem database (https://pubchem.ncbi.nlm.nih.gov/) [14] query has not been included in the TCMSP target compounds in 2D charts, and in SwissTargetPrediction online data platform (http://www.swisstargetprediction.ch/) using the 2D structure predict target compounds. All filtered targets were collected and standardized with the help of Uniprot data platform (https://www.uniprot.org/) [15]. 


\subsection{Predictive targets of functional constipation}

With "functional constipation" as keywords in GeneCards (https://www.genecards.org) and OMIM database (https://omim.org), retrieve the FC may target, The Drugbank database (https://go.drugbank.com/) was used to obtain first-line western drug targets for treatment FC as a supplement [16].The total target of the disease was identified by combining all targets and removing duplicates.

\subsection{Construction of protein interaction network}

Folium sennae, aloe, panax quinquefolium and FC targets to upload Venny 2.1.0 platform (https://bioinfogp.cnb.csic.es/tools/venny/) [17], get targets the intersection of targets of drug targets and disease, three drug combination therapy is the potential targets for FC, the Venn diagram of the relationship between disease targets and drug targets was constructed. Then, the intersection target was uploaded to the String11.0 database (https://string-db.org/) [18], and the species was set as "human" to obtain the protein interaction network. The plug-in cytoNCA of CytoScape3.8.0 was used to analyze the PPI network topology, including degree centrality (DC), betweenness centrality (BC), and eigenvector centrality (EC) and closeness centrality (CC). Firstly, the median of DC nodes is calculated, and all nodes greater than twice the value are screened. Then, the nodes greater than the median value of several other index nodes are screened, which are the key nodes. Then, the String data platform was used to construct the protein interaction network model for the selected key nodes. Then, Cytoscape3.8.0 software was used to perform visual analysis on the protein protein interaction network (PPI network) obtained, and gene ontology (GO) analysis and Kyoto Encyclopedia of Genes and Genomes (KEGG) pathway data were obtained.

\subsection{Target pathway analysis and visualization}

Has a comprehensive comment function Metascape platform (http://metascape.org/gp/index.html), which is not only powerful and update monthly [19], using this data platform, enrichment analysis of GO biological process and enrichment analysis of KEGG pathway was performed for key targets of FC treatment of folium sennae, aloe combined with panax quinquefolium $(\mathrm{P}<0.01)$. Finally using bioinformatics platform (http://www.bioinformatics. com.cn/) [20] with the result of data visualization.

\subsection{Construct the network diagram of "component-target-pathway"}

Cytoscape 3.8.0 software and its built-in tools were used to analyze network topology parameters such as Degree, Betweenness and Closenesss, and to judge the core target and main active ingredients of drug action according to these parameters. At the same time, the network diagram of "component-target-pathway" was constructed.

\subsection{Composition-target molecular docking}

The top 4 targets ranked in degree in the network graph of "component-target-pathway" in the treatment of FC by folium sennae, aloe combined with panax quinquefolium were verified by molecular docking with all the compounds. The three-dimensional structures of target proteins and compounds were obtained from Protein Data Bank (PDB) database (https://www1.rcsb.org/) and TCMSP database. Then AutoDockTools 1.5.6 (http://vina. Scripps.edu/) was used to convert it to PDBQT format [21], and finally Autodock Vina 1.1.2 was used for molecular docking. In order to increase the accuracy of calculation, the parameter exhaustiveness is set to 10, the number of connection modes is 20 , and the binding energy range is 4 .Retain the default values for other parameters. The docking binding energies of 5 proteins and 25 active ingredients were obtained, and the binding strength and activity of the target and compound were evaluated numerically according to the binding energies. The docking results were visually analyzed by pymol 2.4.0 [22].

\section{Results}

\subsection{Collection and screening of components of folium sennae, aloe and panax quinquefolium and their corresponding targets}

Preliminary extraction of chemical components in folium sennae, aloe and panax quinquefolium were 53, 53 and 153, respectively. After screening, 8 active components in folium sennae, 8 in aloe and 10 in Panax quinquefolium were obtained, including quercetin, kaempferol and ginsenoside, as shown in Table 1 . Then the target proteins of the above active compounds were obtained through TCMSP database and SwissTargetPrediction online data platform. Finally, 95, 90 and 63 targets of the active components of folium sennae, aloe and panax quinquinus were 
obtained, and 177 targets were obtained by deleting the duplicate value after combination.

Table 1. Main components of folium sennae, aloe and panax quinquefolium

\begin{tabular}{|c|c|c|c|c|c|}
\hline ID & Mol ID & Molecule Name & OB (\%) & DL & Source \\
\hline MOL1 & MOL000359 & sitosterol & 36.91 & 0.75 & folium sennae and aloe \\
\hline MOL2 & MOL000422 & kaempferol & 41.88 & 0.24 & folium sennae \\
\hline MOL3 & MOL000449 & Stigmasterol & 43.83 & 0.76 & folium sennae \\
\hline MOL4 & MOL002259 & Physciondiglucoside & 41.65 & 0.63 & folium sennae \\
\hline MOL5 & MOL002268 & rhein & 47.07 & 0.28 & folium sennae \\
\hline MOL6 & MOL002288 & Emodin-1-O-beta-D-glucopyranoside & 44.81 & 0.8 & folium sennae \\
\hline MOL7 & MOL002293 & Sennoside D_qt & 61.06 & 0.61 & folium sennae \\
\hline MOL8 & MOL002372 & $\begin{array}{l}\text { (6Z,10E,14E,18E)-2,6,10,15,19,23-hexamethyltetrac } \\
\text { osa-2,6,10,14,18,22-hexaene }\end{array}$ & 33.55 & 0.42 & folium sennae \\
\hline MOL9 & MOL000098 & quercetin & 46.43 & 0.28 & aloe \\
\hline MOL10 & MOL000471 & aloe-emodin & 83.38 & 0.24 & aloe \\
\hline MOL11 & MOL000953 & CLR & 37.87 & 0.68 & aloe \\
\hline MOL12 & MOL001439 & arachidonic acid & 45.57 & 0.2 & aloe \\
\hline MOL13 & MOL002773 & beta-carotene & 37.18 & 0.58 & aloe \\
\hline MOL14 & MOL005043 & campest-5-en-3beta-ol & 37.58 & 0.71 & aloe \\
\hline MOL15 & MOL005051 & Aloeresin C & 34.99 & 0.5 & aloe \\
\hline MOL16 & MOL000358 & beta-sitosterol & 36.91 & 0.75 & panax quinquefolium \\
\hline MOL17 & MOL005344 & ginsenoside rh2 & 36.32 & 0.56 & panax quinquefolium \\
\hline MOL18 & MOL006774 & stigmast-7-enol & 37.42 & 0.75 & panax quinquefolium \\
\hline MOL19 & MOL006980 & papaverine & 64.04 & 0.38 & panax quinquefolium \\
\hline MOL20 & MOL008173 & daucosterol_qt & 36.91 & 0.75 & panax quinquefolium \\
\hline MOL21 & MOL008397 & Daturilin & 50.37 & 0.77 & panax quinquefolium \\
\hline MOL22 & MOL011394 & $\begin{array}{c}\text { (2R,3S,4S,5R,6R)-2-(hydroxymethyl)-6-[[(3S,5R,8R } \\
\text {,9R,10R,12R,13R,14R,17S)-12-hydroxy-4,4,8,10,14- } \\
\text { pentame- } \\
\text { thyl-17-[(2S)-6-methyl-2-[(2S,3R,4S,5S,6R)-3,4,5-tri } \\
\text { hy- } \\
\text { droxy-6-(hydroxymethyl)oxan-2-yl]oxyhept-5-en-2- } \\
\text { yl]-2,3,5,6,7,9,11,12,13,15,16,17-dodecahydro-1H-c }\end{array}$ & 36.43 & 0.25 & panax quinquefolium \\
\hline MOL23 & MOL011435 & PQ-2 & 36.74 & 0.19 & panax quinquefolium \\
\hline MOL24 & MOL011442 & $\begin{array}{l}\text { (8S,9S,10R,13R,14S,17R)-17-[(1R,4R)-4-ethyl-1,5-d } \\
\text { imethylhex- } \\
\text { yl]-10,13-dimethyl-1,2,8,9,11,12,14,15,16,17-decahy } \\
\text { drocyclopenta[a]phenanthren-7-one }\end{array}$ & 43.87 & 0.75 & panax quinquefolium \\
\hline MOL25 & MOL011455 & 20-Hexadecanoylingenol & 32.7 & 0.65 & panax quinquefolium \\
\hline
\end{tabular}

\subsection{Acquisition of FC related targets}

A total of 5,248 functional constipation targets were obtained from Genecards, 25 from OMIM, and 27 from DrugBank database, respectively. Combined with the removal of repeated targets, a total of 5,280 FC related targets were obtained.

\subsection{Establishment and analysis of PPI network}

The screened targets and FC targets of senna, aloe and panax quinquinus were introduced into Venny2.1.0 for analysis, and the Venn diagram was drawn (see Figure 1). 133 intersecting targets of drug targets and disease targets were obtained, of which 12 were common targets of the three drugs, and also the similarities of FC mechanisms of 
the three drugs. 34 were common targets of senna and aloe, which was consistent with the characteristics of compatibility and synergistic application of the two. 27 are the targets of senna leaf acting on FC alone, 38 are the targets of aloe vera acting on FC alone, and 21 are the targets of American ginseng acting on FC alone. The combined use of the three drugs expanded the range of targets for the treatment of the disease, and played a synergistic role.

The STRING 11.0 database was used to analyze the interactions between 133 intersecting targets, and topological analysis was performed using Cytoscape software plug-in cytoNCA. The median values of DC, BC, EC and CC were calculated to be 13, 59.137 21, 0.04351 and 0.458 33, respectively. Based on the 1.3 screening criteria, 31 core targets were obtained, imported into the STRING database, and the results were clarified using Cytoscape software (Figure 2). The closer the target is to the center of concentric circles, the stronger the interaction and the deeper the color. The 14 innermost target proteins, PTGS2, MAPK1, JUN, VEGFA, IL6, TP53 and TNF, are the core action targets of senna leaf and aloe combined with American ginseng in the treatment of FC.

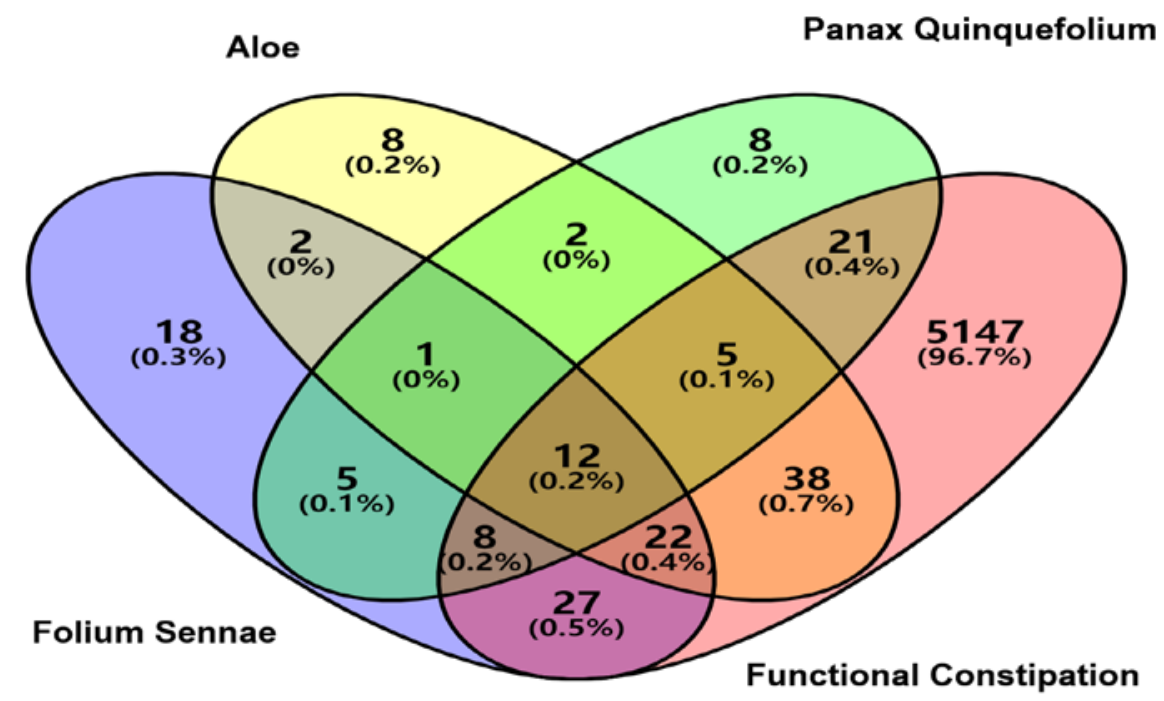

Figure 1. Venny analysis of targets of folium sennae, aloe and panax quinquefolium and functional constipation.

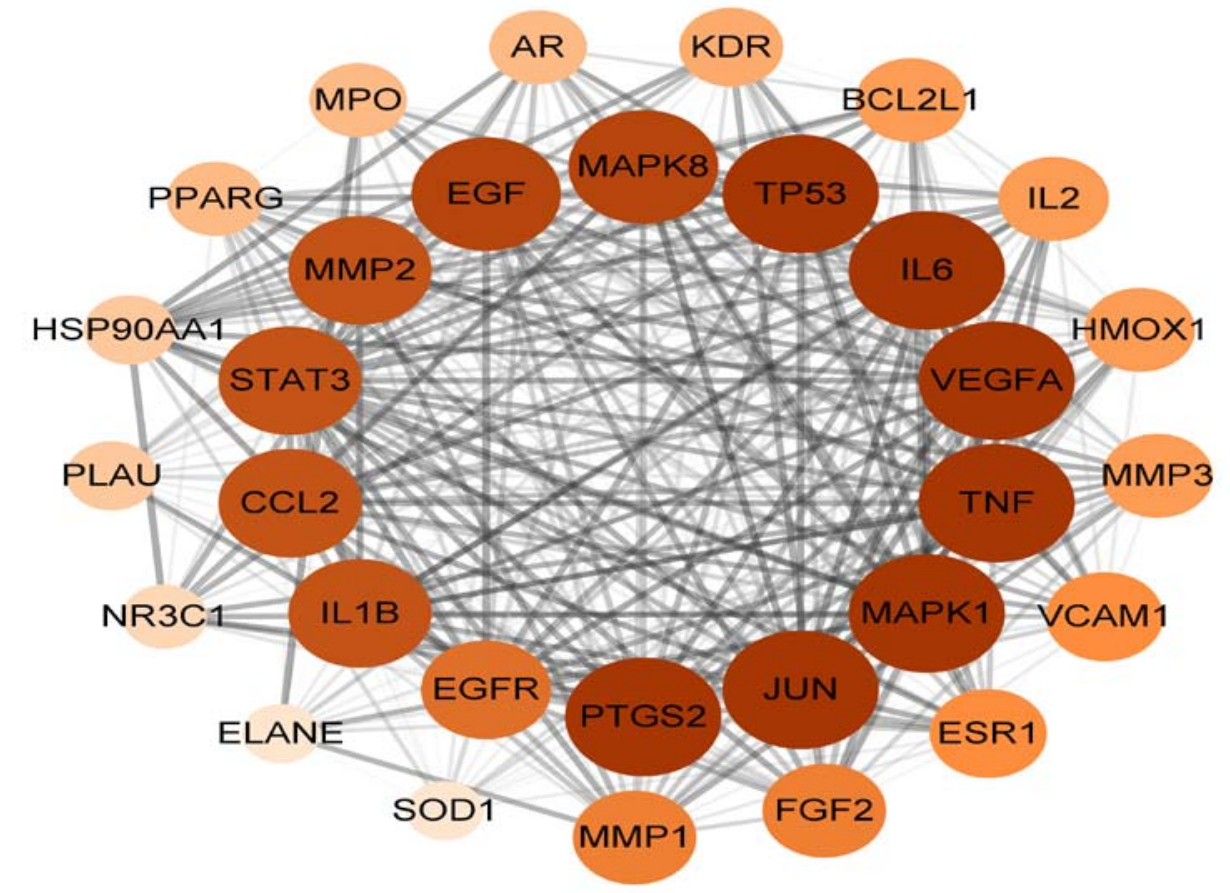

Figure 2. PPI network of folium sennae, aloe combined with panax quinquefolium for FC treatment. 


\subsection{Analysis of enrichment results of GO and KEGG pathways}

Metascape data platform enrichment analysis results showed that 31 core targets of senna and aloe combined with American ginseng in FC treatment were enriched and 201 pathways were obtained, which were involved in 907 biological processes, 53 molecular functions and 29 cell components. The top biological processes in the screening list are: positive regulation of cell migration, cell response to oxidative stress, epithelial cell proliferation, blood vessel development, positive regulation of pri-miRNA transcription by RNA polymerase II, etc., as shown in Figure 3(A). In terms of molecular functions, it mainly involves nuclear receptor activity, cytokine activity, phosphatase binding, integrin binding, serine-type endopeptidase activity, protein domain specific binding, etc., as shown in Figure 3(B). In terms of cell components, it mainly involves: vesicle lumen, membrane raft, RNA polymerase II transcription factor complex, extracellular matrix, mitochondrial matrix, receptor complex, etc., as shown in Figure 3(C).

The enrichment results of KEGG pathway showed that tumors, blood vessels, inflammation, endocrine and other aspects were mainly involved, as shown in Figure 3(D). Including TNF signaling pathway, VEGF signaling pathway, IL-17 signaling pathway, Prolactin signaling Pathway, MAPK signaling pathway, PI3K-Akt signaling pathwayPathway, NF-kB signaling pathway, and the enrichment results of target pathways were shown in Table 2.

A
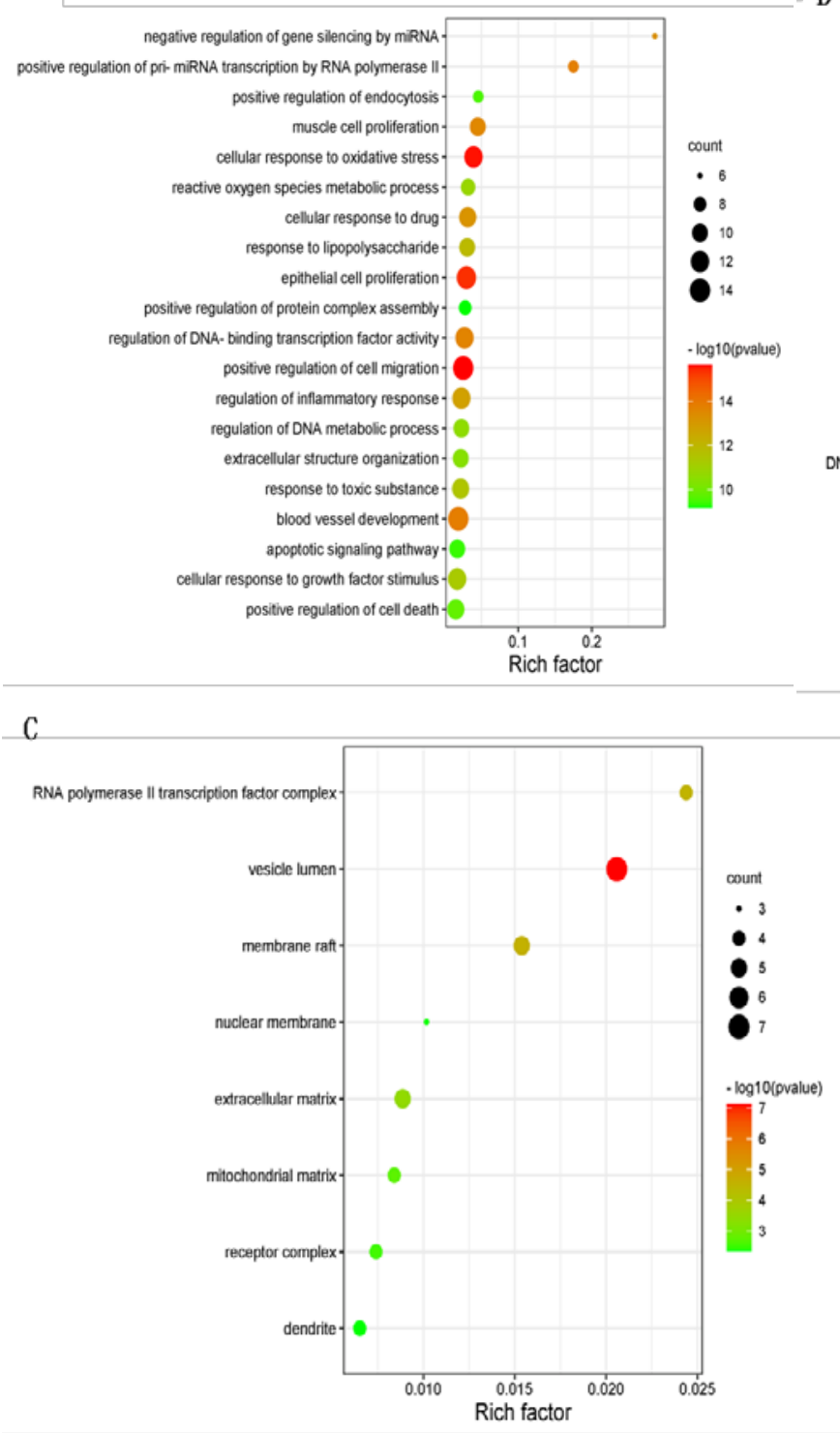

B

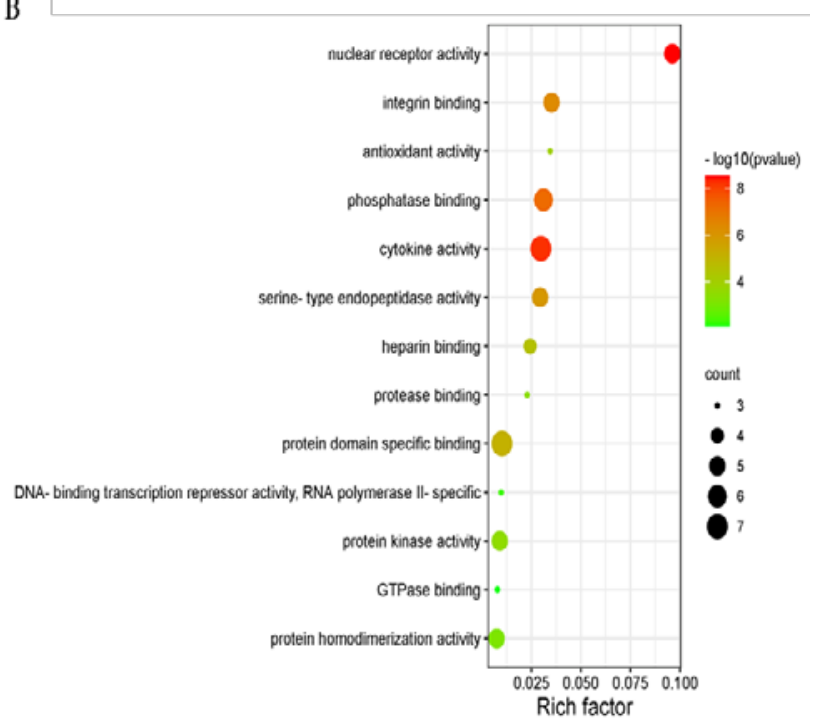

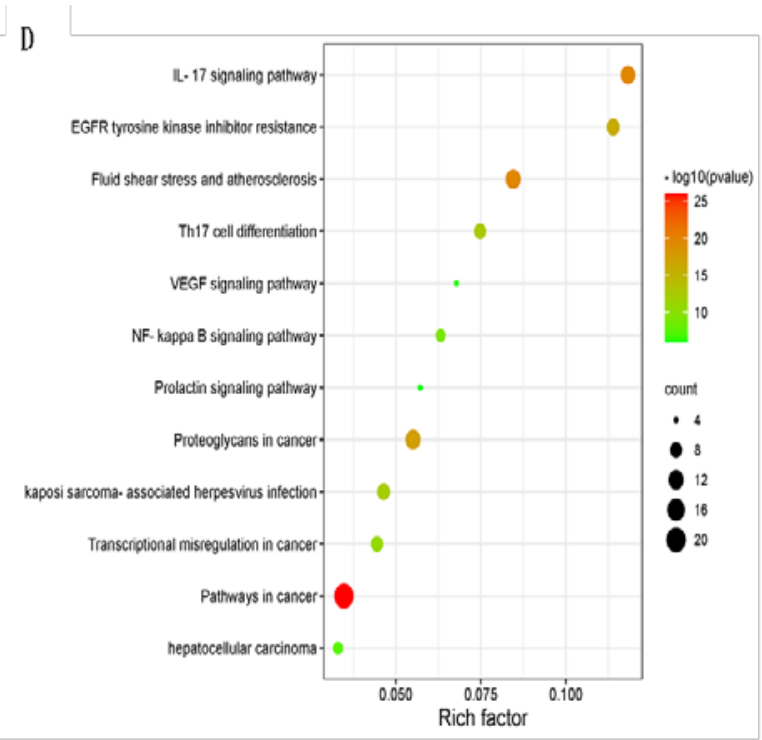

Figure 3. Enrichment analysis of core targets in folium sennae, aloe and panax quinquefolium. 
Table 2. Enrichment results of target pathway of folium sennae, aloe combined with panax quinquefolium in the treatment of FC

\begin{tabular}{|c|c|c|c|c|}
\hline GO & Description & Count & $\log 10(\mathrm{P})$ & Target \\
\hline hsa05200 & Pathways in cancer & 20 & -26.08 & $\begin{array}{c}\text { AR, BCL2L1, EGF, EGFR, ESR1, FGF2, HMOX1, HSP90AA1, IL2, } \\
\text { IL6, JUN, MMP1, MMP2, PPARG, MAPK1, MAPK8, PTGS2, STAT3, } \\
\text { TP53, VEGFA }\end{array}$ \\
\hline ko05418 & $\begin{array}{l}\text { Fluid shear stress } \\
\text { and atherosclerosis }\end{array}$ & 12 & -19.66 & $\begin{array}{l}\text { HMOX1, HSP90AA1, IL1B, JUN, KDR, MMP2, MAPK8, CCL2, TNF, } \\
\text { TP53, VCAM1, VEGFA }\end{array}$ \\
\hline ko04657 & $\begin{array}{l}\text { IL-17 signaling } \\
\text { pathway }\end{array}$ & 11 & -19.65 & $\begin{array}{l}\text { HSP90AA1, IL1B, IL6, JUN, MMP1, MMP3, MAPK1, MAPK8, PTGS2, } \\
\text { CCL2, TNF }\end{array}$ \\
\hline hsa05205 & $\begin{array}{l}\text { Proteoglycans in } \\
\text { cancer }\end{array}$ & 12 & -17.37 & $\begin{array}{l}\text { EGFR, ESR1, FGF2, IL6, KDR, MMP2, PLAU, MAPK1, STAT3, TNF, } \\
\text { TP53, VEGFA }\end{array}$ \\
\hline hsa01521 & $\begin{array}{l}\text { EGFR tyrosine ki- } \\
\text { nase inhibitor resis- } \\
\text { tance }\end{array}$ & 9 & -15.89 & BCL2L1, EGF, EGFR, FGF2, IL6, KDR, MAPK1, STAT3, VEGFA \\
\hline ko04659 & $\begin{array}{l}\text { Th17 cell differen- } \\
\text { tiation }\end{array}$ & 8 & -12.61 & HSP90AA1, IL1B, IL2, IL6, JUN, MAPK1, MAPK8, STAT3 \\
\hline hsa05167 & $\begin{array}{l}\text { kaposi sarco- } \\
\text { ma-associated her- } \\
\text { pesvirus infection }\end{array}$ & 9 & -12.29 & FGF2, IL6, JUN, MAPK1, MAPK8, PTGS2, STAT3, TP53, VEGFA \\
\hline ko05202 & $\begin{array}{l}\text { Transcriptional mi- } \\
\text { sregulation in cancer }\end{array}$ & 8 & -10.78 & BCL2L1, ELANE, IL6, MMP3, MPO, PLAU, PPARG, TP53 \\
\hline ko04064 & $\begin{array}{l}\text { NF-kappa B signal- } \\
\text { ing pathway }\end{array}$ & 6 & -9.06 & BCL2L1, IL1B, PLAU, PTGS2, TNF, VCAM1 \\
\hline hsa05225 & $\begin{array}{l}\text { hepatocellular car- } \\
\text { cinoma }\end{array}$ & 6 & -7.36 & BCL2L1, EGFR, HMOX1, IL6, MAPK1, TP53 \\
\hline ko04370 & $\begin{array}{l}\text { VEGF signaling } \\
\text { pathway }\end{array}$ & 4 & -6.28 & KDR, MAPK1, PTGS2, VEGFA \\
\hline ko04917 & $\begin{array}{c}\text { Prolactin signaling } \\
\text { pathway }\end{array}$ & 4 & -5.98 & ESR1, MAPK1, MAPK8, STAT3 \\
\hline
\end{tabular}

\subsection{Construction of the composite-target-pathway network diagram}

CytoScape 3.8.0 software and its built-in tool Network Analyzer were used to analyze the network topology parameters of FC treated by folium sennae, aloe combined with panax quinquefolium, and a "component-targetpathway" network diagram was constructed, as shown in Figure 4. According to the results of network topology parameters, quercetin is predicted to be the primary active ingredient in the three-drug combination treatment of FC, its Degree is 22, the Betweenness Centrality is 0.2176, and the Closeness Centrality is 0.5377 . Kaempferol, beta-carotene and ginsenoside F2 are the secondary active ingredients, as shown in Table 3.

The Degree of PTGS2 in the network was 16, the Betweenness Centrality was 0.1911, and the Closeness Centrality was 0.5089 . PTGS2 was predicted to be the main target of folium sennae, aloe combined with panax quinquefolium in the treatment of FC, and MAPK1, JUN, VEGFA, IL6, TNF, MAPK8, STAT3, IL1B and so on were also relatively important targets, as shown in Table 2.

\subsection{Molecular docking verification}

The top 4 targets in FC “component-target-pathway" network of folium sennae, aloe combined with panax quinquefolium were selected: PTGS2 (PDB ID:5IKR), MAPK1 (PDB ID:6SLG), JUN (PDB ID:5FV8), VEGFA (PDB ID:4QAF), IL6 (PDB ID:1ALU) were validated by molecular docking with 25 selected active compounds. The results were shown in Table 4 . The lower the binding energy of the ligand and the receptor is, the greater the possibility of interaction is, and the more stable the conformation is. When the binding energy is $<-5 \mathrm{kcal} / \mathrm{mol}$, the 
binding activity is good; when the binding energy is $<-7 \mathrm{kcal} / \mathrm{mol}$, the binding activity is strong [23]. The average binding energies of PTGS2, MAPK1, JUN, VEGFA and IL6 with the selected 25 active compounds were less than $-5 \mathrm{kcal} / \mathrm{mol}$, indicating good binding activity. The top 3 active ingredients with medium value in FC "componenttarget pathway" network graph of folium sennae, aloe combined with panax quinquinae were selected: quercetin (MOL9), kaempferol (MOL2), beta-carotene (MOL13) and ginsenoside F2 (MOL22) for molecular interconnection image display and analysis, as shown in Figure 5.

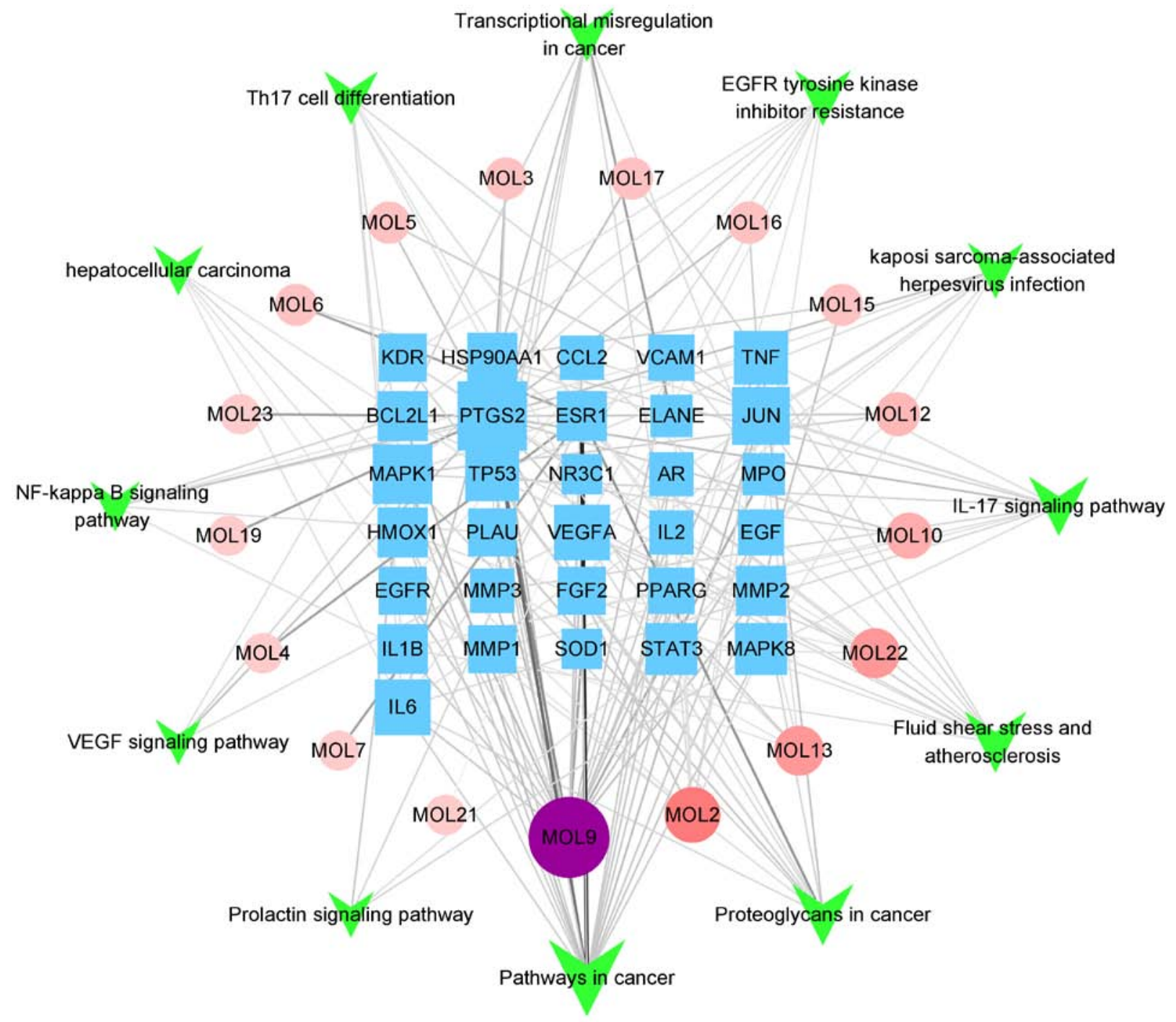

Figure 4. Network diagram of "component-target-pathway" of FC treated by folium sennae, aloe combined with panax quinquefolium.

Table 3. Characteristic parameters of network nodes of main active components in folium sennae, aloe and panax quinquefolium

\begin{tabular}{ccccc}
\hline MOL ID & Molecule Name & Degree & Betweenness Centrality & Closeness Centrality \\
\hline MOL000098 & quercetin & 22 & 0.217505349 & 0.537735849 \\
MOL000422 & kaempferol & 9 & 0.024807572 & 0.413043478 \\
MOL002773 & beta-carotene & 6 & 0.010748836 & 0.395833333 \\
MOL011394 & Ginsenoside F2 & 6 & 0.008696445 & 0.365384615 \\
MOL000471 & aloe-emodin & 4 & 0.005073999 & 0.38 \\
MOL001439 & arachidonic acid & 3 & 0.003556838 & 0.37012987 \\
\hline
\end{tabular}



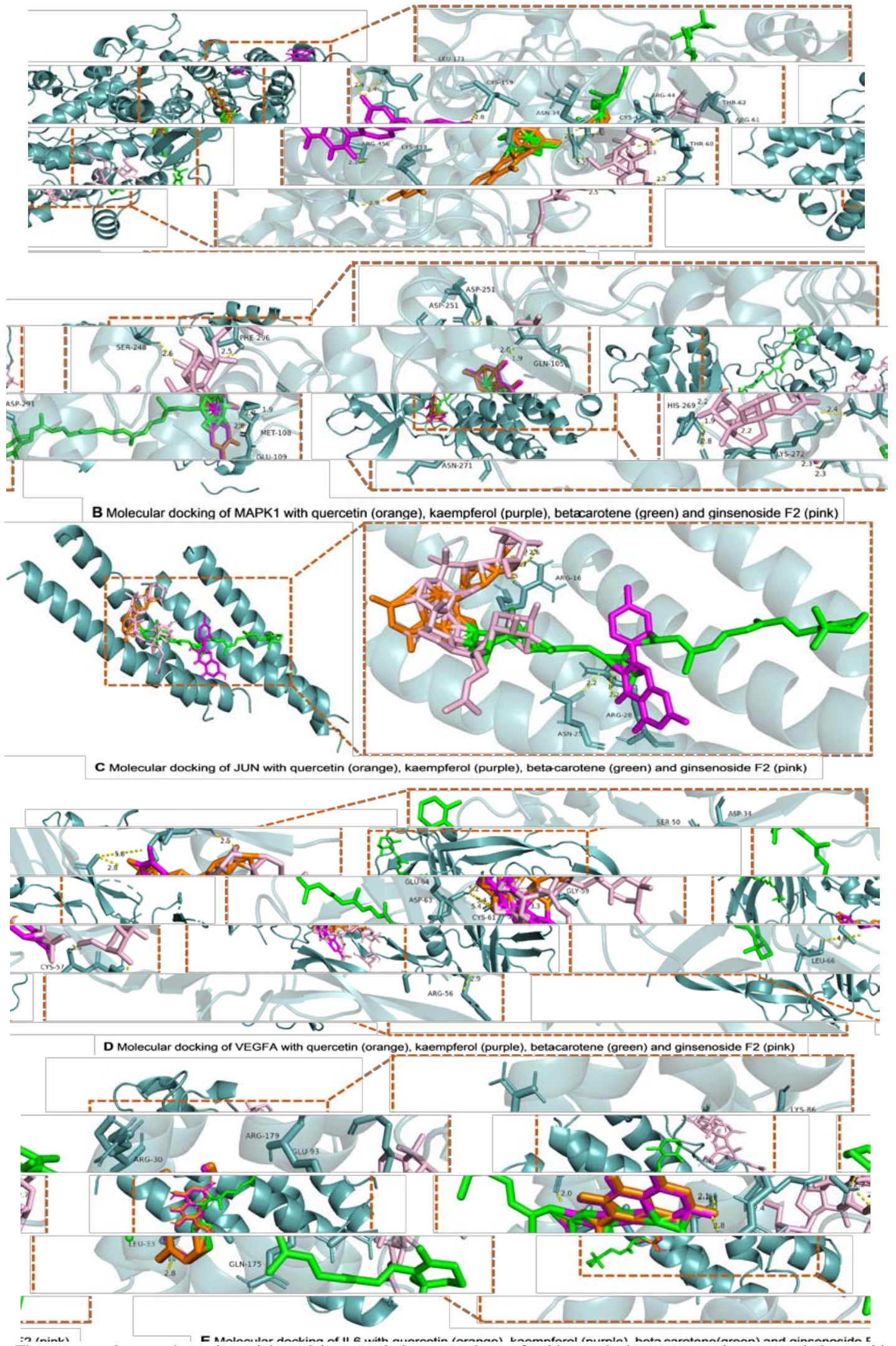

Figure Note: The compounds were shown in a stick model: quercetin in orange, kaempferol in purple, beta-carotene in green and ginsenoside F2 in pink; The protein complex is shown as a blue band model; The yellow dotted lines represent hydrogen bonds.

Figure 5. Schematic diagram of molecular docking. 
Quercetin, kaempferol, beta-carotene and ginsenoside F2 had good binding activity with PTGS2, MAPK1, JUN, VEGFA and IL6, respectively. The average values of the binding energies were $-7.4 \mathrm{kcal} / \mathrm{mol},-7.2 \mathrm{kcal} / \mathrm{mol}$, $-8.8 \mathrm{kcal} / \mathrm{mol}$ and $-7.4 \mathrm{kcal} / \mathrm{mol}$, respectively. In its binding mode, quercetin, kaempferol and ginsenoside F2 had hydrogen bonding with amino acid residues in the five core target proteins, respectively, while beta-carotene did not have hydrogen bonding.

Table 4. The binding energy of active components from folium sennae, aloe and panax quinquefolium with key targets

\begin{tabular}{|c|c|c|c|c|c|c|}
\hline \multirow{2}{*}{ ID } & \multicolumn{6}{|c|}{ binding energy $/\left(\mathrm{kcal} \cdot \mathrm{mol}^{-1}\right)$} \\
\hline & PTGS2 & MAPK1 & JUN & VEGFA & IL6 & Average \\
\hline MOL1 & -8.0 & -8.2 & -6.9 & -7.2 & -7.5 & -7.6 \\
\hline MOL2 & -7.3 & -7.9 & -6.4 & -7.4 & -6.9 & -7.2 \\
\hline MOL3 & -7.5 & -8.3 & -6.8 & -7.8 & -6.6 & -7.4 \\
\hline MOL4 & -10.1 & -9.5 & -7.4 & -8.4 & -7.4 & -8.6 \\
\hline MOL5 & -7.8 & -8.7 & -6.4 & -7.5 & -6.8 & -7.4 \\
\hline MOL6 & -8.3 & -9.4 & -7.1 & -8.0 & -7.0 & -8.3 \\
\hline MOL7 & -8.5 & -8.7 & -8.1 & -7.8 & -7.7 & -8.2 \\
\hline MOL8 & -6.6 & -7.2 & -6.4 & -6.6 & -5.3 & -6.4 \\
\hline MOL9 & -8.1 & -7.9 & -6.4 & -7.5 & -6.9 & -7.4 \\
\hline MOL10 & -9.0 & -8.3 & -6.2 & -6.9 & -6.5 & -7.0 \\
\hline MOL11 & -7.2 & -9.0 & -6.6 & -7.1 & -6.9 & -7.0 \\
\hline MOL12 & -6.2 & -5.6 & -5.2 & -6.6 & -4.7 & -5.5 \\
\hline MOL13 & -10.0 & -8.7 & -8.3 & -10.4 & -7.7 & -8.8 \\
\hline MOL14 & -8.6 & -9.6 & -7.5 & -8.0 & -6.6 & -8.1 \\
\hline MOL15 & -8.0 & -6.2 & -6.7 & -7.2 & -6.8 & -6.7 \\
\hline MOL16 & -8.2 & -9.0 & -7.5 & -7.8 & -6.6 & -7.5 \\
\hline MOL17 & -8.3 & -8.6 & -7.5 & -8.8 & -7.3 & -8.1 \\
\hline MOL18 & -7.6 & -8.2 & -7.2 & -7.4 & -7.8 & -7.6 \\
\hline MOL19 & -8.4 & -7.8 & -6.1 & -7.9 & -6.0 & -7.6 \\
\hline MOL20 & -8.0 & -8.0 & -6.9 & -7.0 & -6.9 & -6.9 \\
\hline MOL21 & -8.7 & -8.6 & -8.0 & -8.6 & -7.5 & -8.4 \\
\hline MOL22 & -8.2 & -7.6 & -6.8 & -7.3 & -7.3 & -7.4 \\
\hline MOL23 & -5.0 & -5.7 & -5.0 & -5.7 & -4.4 & -5.3 \\
\hline MOL24 & -7.4 & -9.2 & -6.5 & -6.5 & -6.8 & -7.3 \\
\hline MOL25 & -7.4 & -7.9 & -6.4 & -7.0 & -6.8 & -7.1 \\
\hline
\end{tabular}

\section{Discussion}

The efficacy of senna and aloe combined with panax quinquefolium in the treatment of functional constipation has been widely recognized. Xu Zhenyuan et al. [24] found that panax quinquefolium could balance intestinal flora in mice with constipation model, promote intestinal peristalsis, improve intestinal motility, and improve intestinal slow transmission state. Panax quinquefolium has a good effect of clearing away heat and producing fluid. When used in proper amount with other purgative drugs, it can play a role of purging heat and defecating without hurting Yin fluid and assisting healthy qi to facilitate large intestine transmission, so it is suitable for patients with chronic constipation [25]. The combined treatment of three drugs for FC has a good laxative effect, its mechanism of action needs to be further explored.

In this study, 25 active components were screened based on the network pharmacology method, among which the core components were quercetin, kaempferol, beta-carotene, ginsenoside F2, etc. The gastrointestinal flora of 
patients with constipation is in an unbalanced state, and various probiotics and strains may have beneficial effects on intestinal motility and constipation [26]. Zhang Jinwei et al. [27] found that quercetin can activate beneficial intestinal flora and inhibit harmful intestinal flora in mice, so as to protect intestinal mucosa and reduce the effects of cytokines on intestinal damage. AaLX et al. [28] showed that kaempferol can balance the intestinal flora and regulate microbial metabolism in mice by oral administration. Beta-carotene is A precursor of vitamin A [29]. Vitamin A-mediated regulation of intestinal epithelium and mucosal immune system jointly forms the intestinal microbiome to maintain the homeostasis of intestinal microbiome [30]. F2 for the original ginsenosides of ginseng glycol type saponins metabolites [31], yan-yan liu, et al. [32], the study found that ginseng saponins can increase mice intestinal fluorescent pseudomonas and butyric acid number of clostridium, in turn, promote the advantage bacterium proliferation, inhibit the growth of pathogenic bacteria in the gut of engraftment and thus improve the health level of mice. According to the literatures reviewed above, quercetin, kaempferol, beta-carotene, ginsenoside F2 and other active ingredients play a role in regulating constipation by regulating intestinal flora, promoting the growth of dominant flora and other mechanisms. Molecular docking validation results also showed that the above active ingredients had strong binding activities with the core targets (PTGS2, MAPK1, JUN, VEGFA, IL6). Therefore, the treatment of FC by folium sennae, aloe combined with panax quinquefolium may be mainly achieved by acting on quercetin, kaempferol, beta-carotene, ginsenoside F2 and other active ingredients.

The results showed that five core targets, PTGS2, MAPK1, JUN, VEGFA and IL6, played a major role in the network map. Prostaglandin (PG) H synthase exists as two subtypes, called PGH synthase -1 and -2, which are encoded by Ptgs1 and Ptgs2 genes and commonly known as cox-1 and COX-2 [33]. Studies have shown that COX-2 is involved in the occurrence of intestinal motor dysfunction after intestinal obstruction. When intestinal obstruction is proximal dilated, the contractility of periintestinal smooth muscle is significantly decreased, and the expression of COX-2 mRNA and protein is significantly up-regulated. The use of COX-2 inhibitor NS-398 can significantly improve the contractility of colonic smooth muscle cells in the model rats of colonic obstruction. Dilate the obstruction lumen [34]. MAPK is a complex pathway composed of multiple proteins [35], which can regulate the expression of AQP4 [36]. The expression of AQP3 and AQP4 in colon tissues of constipation model rats is significantly increased, which is also one of the mechanisms of STC. AQP3 and AQP4 can be down-regulated through MAPKs signaling pathway, thus reducing intestinal water reabsorption. Increase fecal water content [37]. C-jun is the main component of dimer transcription factor activator protein-1 (AP-1) [38], li Boqing et al. [39] demonstrated that the expression of AP-1 increased in the colon tissues of model mice with intestinal flora imbalance. VEGFA can relax the tight connections between intestinal cells, thus destroying the intestinal mucosal barrier and causing intestinal microorganisms to invade the intestinal wall [40]. Il-6 promotes intestinal mucosal inflammatory infiltration by affecting intestinal mucosal CD4 ( + ) T cells [41]. Exogenous IL-6 can directly promote colon contraction in rats, activate gastrointestinal submucosal neurons and participate in the regulation of gastrointestinal function, affect adrenal stress response and gastric peristalsis, and lead to gastrointestinal motility and secretion dysfunction [42]. These targets involve cell migration, oxidative stress, epithelial cell proliferation, vascular development, inflammation, reactive oxygen metabolism and other biological processes, including cytokines, proteins, enzymes, transcription factors, etc., and are the core substances of Senna, aloe vera combined with panax quinquefolium in the treatment of FC.

According to the enrichment analysis of KEGG pathway, senna and aloe combined with panax quinquefolium treated FC by regulating tumor necrosis factor, MAPK, PI3K-Akt, NF-kB and other signaling pathways. Studies have shown that intrabdominal injection of TNF $\alpha$ in mice can significantly damage the cell network structure of Cajal mesenchymal cells [43], and Cajal mesenchymal cells are an important part of the gastrointestinal motility regulation unit and can promote intestinal transport [44]. Wan Ye min et al. [45] showed that regulating the expression of MPKA signaling pathway can increase intestinal water and promote intestinal peristalsis in model rats.PI3K/Akt signaling pathway plays an important role in signal transduction related to cell division, proliferation, movement, and metabolism [46], and participates in the pathogenesis of FC by affecting the function of distal colon tissue [47]. Jiang Z. C. and Huang S. X. [48] showed that the expression of NF- $\kappa$ B protein and its mRNA in constipation model rats was significantly increased, indicating that NF- $\mathrm{B}$ signaling pathway is closely related to the pathogenesis of constipation. Other FC related pathways, such as interleukin-17 signaling pathway, vascular endothelial growth factor signaling pathway and prolactin signaling pathway, were enriched in KEGG analysis in this study, indicating that Senna and aloe combined with panax quinquefolium play a role in the treatment of functional constipation by regulating multiple signaling pathways.

The results show that panax quinquefolium joint senna, aloe synergies role in the treatment of functional constipation, panax quinquefolium tonifying qi, nourishing Yin, oneself clear heat, can make up for the senna, the aloe 
vera in tianjin, the waste gas with tianjin disadvantage, at the same time, the main active ingredients of ginseng American ginseng saponin F2, saponins rh2 of ginseng has a regulation, and balance the role of the intestinal flora, other active components and core targets have high degree values in the network diagram. In addition, the enrichment analysis of KEGG pathway and molecular docking results also showed that panax quinquinosa plays an important role in the treatment of functional constipation in combination with Senna and aloe, which reflects that the combined use of the three drugs has the characteristics of multi-component, multi-target and multi-pathway, and the important targets and pathways are closely linked and unified. Through the analysis and verification of key components, key targets and important pathways in relevant literature, the specific mechanism of the treatment of FC by senna leaf and aloe combined with American ginseng was preliminaries understood, which provided ideas for clinical drug use and laid a foundation for further in-depth discussion of its mechanism of action. However, due to the limitation of network pharmacology methodology and the difference between TCM syndrome differentiation theory and modern medical thinking, animal experiments should be carried out to verify relevant targets and pathways.

\section{References}

[1] Wang Dongdong, Wu Xiangbai. (2019). Chinese Journal of General Practice, 2019, 22(24): 3016-3022.

[2] Aziz, I., Whitehead, W. E., Palsson, O. S., Törnblom, H., Simrén, M. (2020). An approach to the diagnosis and management of Rome IV functional disorders of chronic constipation. Expert Rev Gastroenterol Hepatol, 2020 Jan, 14(1): 39-46. doi: 10.1080/17474124.2020.1708718. Epub 2020 Jan 2. PMID: 31893959.

[3] Koppen, I. J. N., Vriesman, M. H., Saps, M., Rajindrajith, S., Shi, X., van Etten-Jamaludin, F. S., Di Lorenzo, C., Benninga, M. A., Tabbers, M. M. (2018). Prevalence of Functional Defecation Disorders in Children: A Systematic Review and Meta-Analysis. J Pediatr., 2018 Jul, 198: 121-130. e6.doi: 10.1016/j.jpeds.2018.02.029. Epub 2018 Apr 12. PMID: 29656863.

[4] Suares, N. C., Ford, A. C. (2011). Prevalence of, and risk factors for, chronic idiopathic constipation in the community: systematic review and meta-analysis. Am J Gastroenterol, 2011Sep, 106(9): 1582-91; quiz1581, 1592. doi: 10.1038/ajg.2011.164. Epub 2011 May 24. PMID: 21606976.

[5] Tang Weifeng, Tang Xiaojun, Yang Wei. (2015). Research progress of Chinese and western medicine on functional constipation [J]. World Journal of Integrated Chinese and Western Medicine, 2015, 10(06): 880-884.

[6] Yuan Meng chun. Clinical research progress of functional constipation [J]. Inner Mongolia Journal of Traditional Chinese Medicine, 40(02): 140-143.

[7] Huang Suwei, Wang Chuijie. (2019). Journal of Liaoning University of Traditional Chinese Medicine, 2019, 21(01): $221-224$.

[8] National Pharmacopoeia Commission. Chinese Pharmacopoeia [M]. Beijing: China Medical Science and Technology Press, 2020.

[9] Yue Wuyang, Peng Zihao, Chen Xuxi, Wu Rui, Chen Jinyao, Zhang Lishi. (2020). Effects of aloe vera, Panax quintillae and Senna leaf on intestinal and defecation function in mice [J]. Modern Preventive Medicine, 2020, 47(08): 1460-1463+1469.

[10] Chen Xuxi, Li Na, Yue Wuyang, Peng Zihao, Wu Rui, Chen Jinyao, Zhang Lishi. (2020). Effects of aloe vera, American ginseng and senna leaf on intestinal microflora in mice with constipation [J]. Modern Preventive Medicine, 2020, 47(24): 4497-4502+4506.

[11] Liu Jiahui, Lu Dongyong, Zhou Houming, Kuang Weihong, Chen Zexiong, Zhang Shijun. (2020). Chinese Journal of Traditional Chinese Medicine, 2020, 45(01): 163-168. (in Chinese)

[12] Liang Hong-bao, Li Rui, Yao Jing-chun, Qin Guo-fei, Zhang Hao, Zhang Gui-min. Study on the mechanism of Action of Shouhui Tongfu Capsule in treating constipation based on network pharmacology and molecular docking method [J]. Chinese Journal of Traditional Chinese Medicine, 46(03): 511-519.

[13] Jinlong Ru, Peng Li, JinanWang, Wei Zhou, Bohui Li, Chao Huang, Pidong Li, Zihu Guo, Weiyang Tao, Yinfeng Yang, Xue $\mathrm{Xu}$, Yan Li, Yonghua Wang, Ling Yang. (2014). TCMSP: a database of systems pharmacology for drug discovery from herbal medicines. J Cheminformatics, 2014 Apr 16, 6(1): 13.

[14] Kim, S., Chen, J., Cheng, T., et al. (2021). PubChem in 2021: new data content and improved web interfaces. Nucleic Acids Res., 2021, 49(D1): D1388-D1395. doi:10.1093/nar/gkaa971.

[15] The UniProt Consortium, UniProt: the universal protein knowledgebase in 2021, Nucleic Acids Research, Volume 49, Issue D1, 8 January 2021, pp. D480-D48.

[16] Wishart, D. S., Feunang, Y. D., Guo, A. C., Lo, E. J., Marcu, A., Grant, J. R., Sajed, T., Johnson, D., Li, C., Sayeeda, Z., Assempour, N., Iynkkaran, I., Liu, Y., Maciejewski, A., Gale, N., Wilson, A., Chin, L., Cummings, R., Le, D., Pon, A., Knox, C., Wilson, M. (2017). DrugBank 5.0: a major update to the DrugBank database for 2018. Nucleic Acids Res., 2017 Nov 8. doi: 10.1093/nar/gkx1037.

[17] Oliveros, J. C. (2007-2015). Venny. An interactive tool for comparing lists with Venn’s diagrams.

[18] STRINGv 11: protein-protein association networks with increased coverage, supporting functional discovery in genome-wide 
experimental datasets. Nucleic Acids Res., 2019 Jan, 47: D607-613.

[19] Zhou, Y., Zhou, B., Pache, L., Chang, M., Khodabakhshi, A. H., Tanaseichuk, O., Benner, C., Chanda, S. K. (2019). Metascape provides a biologist-oriented resource for the analysis of systems-level datasets. Nat Commun., 2019 Apr 3, 10(1): 1523. doi: 10.1038/s41467-019-09234-6. PMID: 30944313; PMCID: PMC6447622.

[20] Heatmap was plotted by an online platform for data analysis and visualization. http://www.bioinformatics.com.cn.

[21] O. Trott, A. J. Olson. (2010). AutoDock Vina: improving the speed and accuracy of docking with a new scoring function, efficient optimization and multithreading. Journal of Computational Chemistry, 31(2010), 455-461.

[22] The PyMOL Molecular Graphics System, Version 2.4.0 Schrödinger, LLC.

[23] Xu Jiahui, Chen Qingguang, Zhang Liqiong, Han Xu, Liu Yahua, Jin Shenyi, Cai Mengjie, Liu Yuying, Lu Hao. Study on the mechanism of astragalus membranaceus in the treatment of Hashimoto's thyroiditis based on network pharmacology and molecular docking method [J]. Shanghai Journal of Traditional Chinese Medicine, 55(04): 6-14.

[24] Xu, Z. Y. (2020). Experimental study on regulating intestinal flora and improving slow transit constipation by Yangyin Yiqi Runchang Recipe [D]. Yunnan University of Chinese Medicine, 2020.

[25] Zou Xian-gang, Fu Shu-jie, Huang Xing-zhen, Wu Ling-ling, Jiang Wei-zhe. (2014). Study on the effect of shijiejiangzhi capsule on moistening intestine and relieving constipation [J]. Shizhen National Medicine and National Medicine, 2014, 25(01): 65-68.

[26] Dimidi, E., Christodoulides, S., Scott, S. M., Whelan, K. (2017). Mechanisms of Action of Probiotics and the Gastrointestinal Microbiota on Gut Motility and Constipation. Adv Nutr., 2017 May 15, 8(3): 484-494. doi:10.3945/an.116.014407. PMID:28507013; PMCID: PMC5421123.

[27] Zhang, J. W. (2014). Study on the purgative mechanism of quercetin and optimization of extraction process of quercetin from pine needles [D]. Guangzhou Medical University, 2014.

[28] Aa, L. X., Fei, F., Qi, Q., Sun, R. B., Gu, S. H., Di, Z. Z., Aa, J. Y., Wang, G. J., Liu, C. X. (2020). Rebalancing of the gut flora and microbial metabolism is responsible for the anti-arthritis effect of kaempferol. Acta Pharmacol Sin., 2020 Jan, 41(1): 73-81. doi: 10.1038/s41401-019-0279-8. Epub 2019 Aug 19. PMID: 31427695; PMCID: PMC7468310.

[29] Krinsky, N. I., Johnsone, J. (2005). Carotenoid actions and their relation to health and disease [J]. Molecular Aspects of Medicine, 2005, 26(6): 459-516.

[30] Cantorna, M. T., Snyder, L., Arora, J. (2019). Vitamin A and vitamin D regulate the microbial complexity, barrier function, and the mucosal immune responses to ensure intestinal homeostasis. Crit Rev Biochem Mol Biol., 2019 Apr, 54(2): 184-192. doi: 10.1080/10409238.2019.1611734. Epub 2019 May 14. PMID: 31084433; PMCID: PMC6629036.

[31] Gao Xia, Geng Ting, Ma Yang, Li Yan-jing, Huang Wen-zhe, Wang Zhen-zhong, Xiao Wei. (2016). Chinese Journal of Traditional Chinese Medicine, 2016, 41(12): 2329-2338.

[32] Liu Yan-yan, Zhang Kai, Guan Jiawei, Cao Xue-jiao, Wu Da-chang. (2015). Effects of ginsenosides on intestinal microflora of BALB/c mice [J]. Advances in Modern Biomedicine, 2015, 15(06): 1041-1045.

[33] Smith, W. L., DeWitt, D. L., Garavito, R. M. (2000). Cyclooxygenases: structural, cellular, and molecular biology. Annu Rev Biochem, 2000, 69: 145-82. doi: 10.1146/annurev.biochem.69.1.145. PMID: 10966456.

[34] Lin, Y. M., Sarna, S. K., Shi, X. Z. (2012). Prophylactic and therapeutic benefits of COX-2 inhibitor on motility dysfunction in bowel obstruction: roles of $\mathrm{PGE}_{2}$ and EP receptors. Am J Physiol Gastrointest Liver Physiol, 2012 Jan 15, 302(2): G267-75. doi: 10.1152/ajpgi.00326.2011. Epub 2011 Oct 28. PMID: 22038825; PMCID: PMC3341114.

[35] Liang, Y. J., Yang, W. X. (2019). Kinesins in MAPK cascade: How kinesin motors are involved in the MAPK pathway? Gene, 2019 Feb 5, 684: 1-9. doi: 10.1016/j.gene.2018.10.042. Epub 2018 Oct 17. PMID: 30342167.

[36] Ma Xiaoyan, Shen Qi, Hua Ying, Xie Ailan, Zhu Xueqiong. (2013). The role of MAPK signaling pathway in regulating AQP3 expression in human amniotic epithelial cells by compound Salvia miltiorrhiza injection [J]. Chinese Journal of Integrated Traditional and Western Medicine, 2013, 33(06): 778-782.

[37] Yang Xiao. (2018). Study on the mechanism of Tongzhan Decoction in the treatment of slow transit constipation based on MAPK signaling pathway [D]. Nanjing University of Chinese Medicine, 2018.

[38] Papavassiliou, A. G., Musti, A. M. (2020). The Multifaceted Output of c-Jun Biological Activity: Focus at the Junction of CD8 T Cell Activation and Exhaustion. Cells, 2020 Nov 13, 9(11): 2470. doi:10.3390/cells9112470. PMID: 33202877; PMCID: PMC7697663.

[39] Li, B., Ding, Y., Cheng, X., Sheng, D., Xu, Z., Rong, Q., Wu, Y., Zhao, H., Ji, X., Zhang, Y. (2020). Polyethylene microplastics affect the distribution of gut microbiota and inflammation development in mice. Chemosphere, 2020 Apr, 244: 125492. doi: 10.1016/j.chemosphere.2019.125492. Epub 2019 Nov 27. PMID: 31809927.

[40] Frysz-Naglak, D., Fryc, B., Klimacka-Nawrot, E., Mazurek, U., Suchecka, W., Kajor, M., Kurek, J., Stadnicki, A. (2011). Expression, localization and systemic concentration of vascular endothelial growth factor (VEGF) and its receptors in patients with ulcerative colitis. Int Immunopharmacol, 2011 Feb, 11(2): 220-5. doi: 10.1016/j.intimp.2010.11.023. Epub 2010 Nov 27. PMID: 21115119. 
[41] Hurst, R. J., De Caul, A., Little, M. C., Kagechika, H., Else, K. J. (2013). The retinoic acid receptor agonist Am80 increases mucosal inflammation in an IL-6 dependent manner during Trichuris muris infection. J Clin Immunol., 2013 Nov, 33(8): 1386-94. doi: 10.1007/s10875-013-9936-8. Epub 2013 Sep 15. PMID: 24036839; PMCID: PMC3825562.

[42] O'Malley, D., Dinan, T. G., Cryan, J. F. (2011). Altered expression and secretion of colonic interleukin-6 in a stress-sensitive animal model of brain-gut axis dysfunction. J Neuroimmunol, 2011 Jun, 235(1-2): 48-55. doi: 10.1016/j.jneuroim.2011.04.003. Epub 2011 May 11. PMID: 21565410.

[43] Zheng Hongqun, Shang Mingfeng, Shen Weidong. (2016). Effects of intraperitoneal injection of tumor necrosis factor $\alpha$ on intestinal Cajal mesenchymal cells in rats [J]. Journal of Harbin Medical University, 2016, 50(05): 393-396+402.

[44] Bernardini, N., Ippolito, C., Segnani, C., Mattii, L., Bassotti, G., Villanacci, V., Blandizzi, C., Dolfi, A. (2013). Histopathology in gastrointestinal neuromuscular diseases: methodological and ontological issues. Adv Anat Pathol, 2013 Jan, 20(1): 17-31. doi: 10.1097/PAP.0b013e31827b65c0. PMID: 23232568.

[45] Wan Ye-min, Zeng Li, Qian Hai-hua. (2019). Effect of Tongzhan Decoction on PKA/MPKA signaling pathway in colon tissue of STC rat model [J]. Chinese Journal of Experimental Formulae, 2019, 25(05): 135-142.

[46] Bader, A. G., Kang, S., Zhao, L., Vogt, P. K. (2005). Oncogenic PI3K deregulates transcription and translation. Nat Rev Cancer, 2005 Dec, 5(12): 921-9. doi: 10.1038/nrc1753. PMID: 16341083.

[47] Li, M. H. (2015). Role of PI3K/AKT/eNOS signaling pathway in slow transit constipation in rats [D]. North China University of Science and Technology, 2015.

[48] Jiang Zhichao, Daffodil. (2018). Journal of Guangxi University of Traditional Chinese Medicine, 2018, 21(01): 1-4. (in Chinese). 